\title{
Biphasic Time Course of Inactivation of Potassium Contractures in Single Twitch Muscle Fibers of the Frog
}

\author{
Torao Nagai, Masa TAKauji, Isao KosaKa, \\ and Masaaki TSUTSU-URA \\ Department of Physiology, Sapporo Medical College, Sapporo, 060 Japan
}

\begin{abstract}
The time course of the inhibition of peak tension (inactivation) of potassium contracture induced by conditioning depolarization with $10,15,20$ and $30 \mathrm{mM} \mathrm{K}^{+}$was examined, using single twitch fibers from the frog semitendinosus muscle. The time-dependence curve of the inactivation was biphasic, consisting of the first phase and the second phase. The first phase was characterized as follows: 1) the time course and the extent depend on the degree of conditioning depolarization; 2) the inactivation proceeds exponentially and finally reaches a steady level; and 3) the rate is markedly increased by lowering $\mathrm{Ca}^{2+}$ in the external medium. It could be considered that the first phase of the inactivation is an analogical phenomenon with the inactivation of sodium conductance in a squid giant axon. The second phase of the inactivation induced by conditioning with 20 and $30 \mathrm{mM} \mathrm{K} \mathrm{K}^{+}$reached its full inactivation independently of the degree of conditioning depolarization. On the basis of these results, the terms inactivation 1 and inactivation 2 were given to the first phase and second phase, respectively, of the time-dependence curve of the inactivation of potassium contracture.
\end{abstract}

We have recently reported that two distinct processes of the inactivation of potassium contracture ( $\mathrm{K}$ contracture) are involved in the inactivation induced by conditioning in $\mathrm{Ca}^{2+}$-free medium, by conditioning depolarization with a subthreshold concentration of $\mathrm{K}$ or by the conditioning depolarization in the presence of dantrolene or other agents (KosAKA et al., 1977; TAKAUJI and NAGAI, 1977). We tentatively termed these two processes inactivation 1 and inactivation 2: inactivation 1 was related to only the spontaneous relaxation of $\mathrm{K}$ contracture, while inactivation 2 was related to only the inhibition of the peak contracture tension in the whole range of the time-dependence curve of the inactivation.

On the other hand, we found that the time course of the inactivation of $\mathrm{K}$ contracture induced by conditioning depolarization is biphasic (TAKAUJI and

Received for publication March 15, 1979

永井寅男, 高氏 昌, 小坂 功, 筒浦理正 
NAGAI, 1977): one phase in which the peak tension of test $\mathrm{K}$ contracture decreases with increasing the time of conditioning and finally reaches a steady level, and the other phase in which the peak tension of the test $\mathrm{K}$ contracture falls with time towards a full inactivation.

In the present paper, the nature of the biphasic time-dependence curve of the inactivation of $\mathrm{K}$ contracture was further investigated. The first and second phases of the curve were characterized and termed inactivation 1 and inactivation 2 , respectively. In addition, results on the mechanical inactivation reported by several investigators were discussed from the points of view obtained in the present studies. This work has been presented at the 6th International Biophysics Congress, Kyoto, Japan (TAKAUJI et al., 1978).

\section{MATERIALS AND METHODS}

Single muscle fibers dissected from the semitendinosus muscles of the frog, Rana japonica, were used.

A single fiber was isolated carefully with a piece of a razor blade and a forceps in Ringer solution under a binocular microscope and was kept in the solution for $30 \mathrm{~min}$ or more, and then the experiment was started if the fiber was intact. The tendon at one end of a fiber was attached to the lever of a strain gauge (Nihon Kohden, SB-1T-H) with chemical cement, Aron Alpha (Toa Gosei Chemical Co.) and the other tendon was fixed on a glass hook attached to a micro-manipulator. The fiber length was adjusted to 1.2 times its slack length by the micro-manipulator. The mechanical responses were recorded on an ink-writing oscillograph (Nihon Kohden, WI-130 or WI-180).

For determining the inactivation of $\mathrm{K}$ contracture, we adopted the procedure primarily used by FRANKENHAEUSER and LÄNNERGREN (1967): before the application of test solution ( $190 \mathrm{~mm} \mathrm{~K}^{+}$Ringer solution), fibers were treated with choline Ringer solution containing various concentrations of $\mathrm{K}^{+}$(conditioning $\mathrm{K}$ solution) for a given period of time. Drawing the time-dependence curve of inactivation of $\mathrm{K}$ contracture, we plotted the relative peak tension of test $\mathrm{K}$ contracture to control contracture against the time given on the abscissa. All of these experiments were performed in the absence of $\mathrm{Na}^{+}$, to inhibit the firing response generated by $\mathrm{K}$ depolarization.

The method of the exchange of the solution followed the description of HodGKIN and Horowicz (1959). The capacity of the experimental chamber was about $0.8 \mathrm{ml}$. The exchange of the solution was performed within a fraction of a second.

Experiments were carried out at room temperature $\left(18-24^{\circ} \mathrm{C}\right)$ and between October, 1977 and March, 1978.

The following solutions were used: Normal Ringer solution containing 110 or $115 \mathrm{~mm} \mathrm{NaCl}, 2.5 \mathrm{~mm} \mathrm{KCl}, 1.8 \mathrm{mM} \mathrm{CaCl}$ and $5 \mathrm{~mm}$ Tris- $\mathrm{HCl}$ buffer (tris 
(hydroxymethyl) aminomethane- $\mathrm{HCl}$ buffer) (pH 7.2). Choline Ringer solution was prepared by replacing all $\mathrm{NaCl}$ in normal Ringer solution with choline chloride. Choline Ringer solution of $0.18 \mathrm{mM} \mathrm{Ca}$ and $10 \mathrm{~mm} \mathrm{~K} \mathrm{~K}^{+}$choline Ringer solution of $0.18 \mathrm{~mm} \mathrm{Ca}$ were also used. Potassium Ringer solution $\left(190 \mathrm{mM} \mathrm{K}^{+}\right)$was prepared by replacing all $\mathrm{NaCl}$ and $\mathrm{KCl}$ in normal Ringer solution with $95 \mathrm{mM} \mathrm{K}_{2} \mathrm{SO}_{4}$.

All chemicals were commercial products of the best reagent grade available.

\section{RESULTS}

Each of the following experimental results is a representative one obtained in several such experiments.

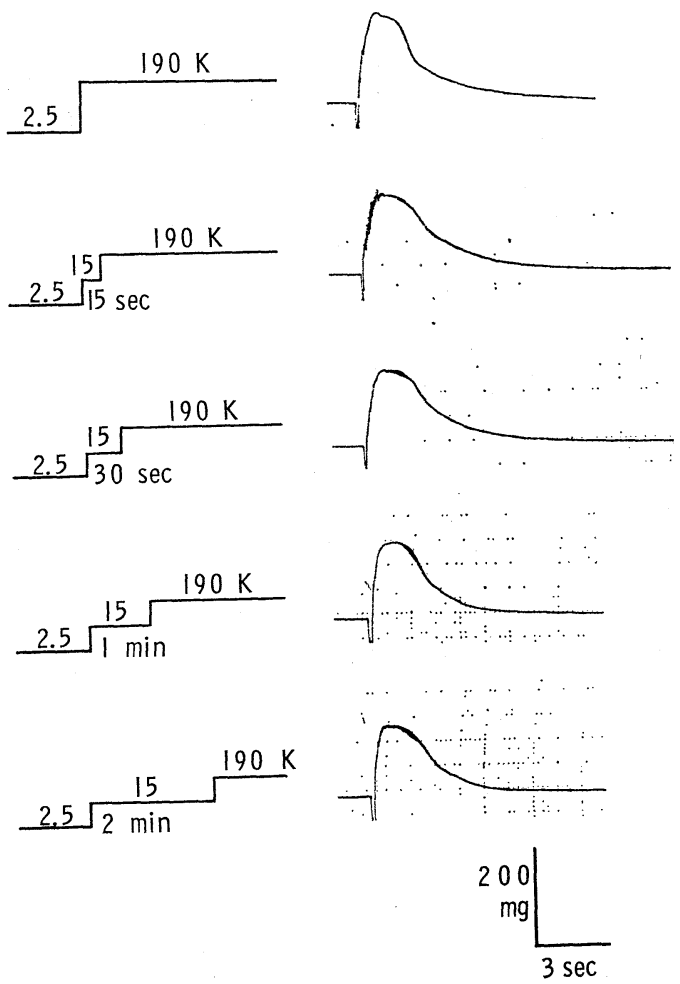

Fig. 1a. The decrease in peak tension of test $\mathrm{K}$ contracture induced by lengthening the duration of conditioning depolarization with $15 \mathrm{mM} \mathrm{K}^{+}$. The sudden downward deflection in each record marks the introduction of high concentration $\mathrm{K}^{+}$solution. Fiber diameter: $90 \mu \mathrm{m}$. Test $\mathrm{K}^{+}$solution: $95 \mathrm{mM} \mathrm{K} \mathrm{KO}_{4}, 1.8 \mathrm{mM} \mathrm{CaCl}_{2}$ and $5 \mathrm{~mm}$ Tris-HCl buffer ( $\mathrm{pH}$ 7.2). Conditioning solution: choline Ringer solution containing $15 \mathrm{~mm} \mathrm{KCl}$. Other conditions and experimental procedures are described in the text. 


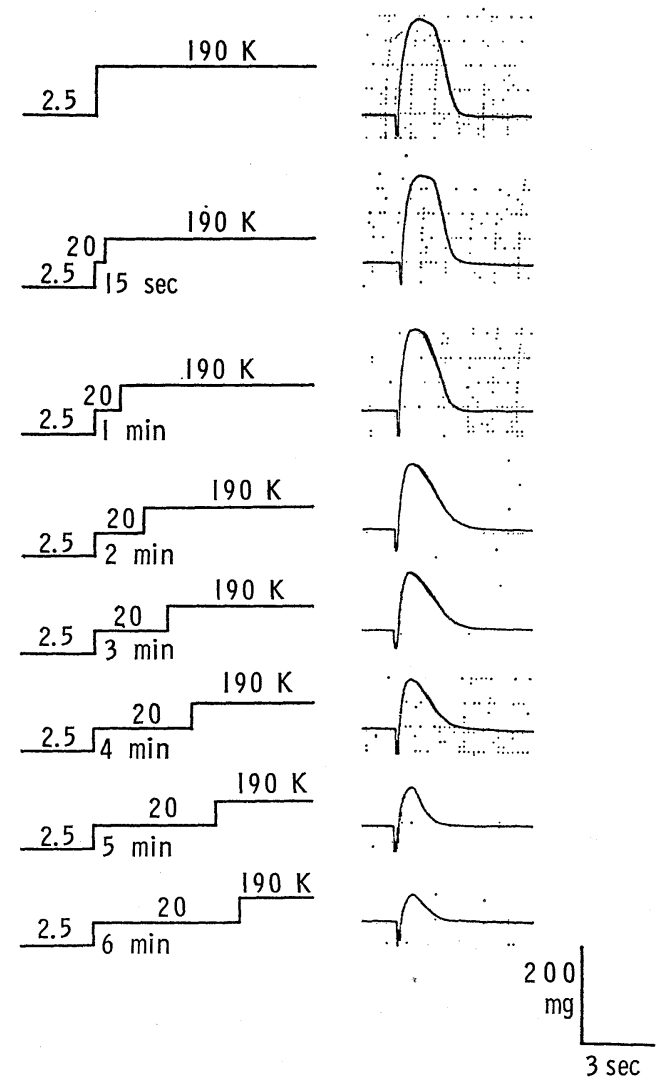

Fig. 1b. The decrease in peak tension of test $\mathrm{K}$ contracture induced by conditioning with $20 \mathrm{~mm} \mathrm{~K} \mathrm{~K}^{+}$. Conditioning solution: choline Ringer solution containing $20 \mathrm{mM} \mathrm{KCl}$. Other conditions are the same as stated for Fig. 1a.

1. Biphasic time course of the inactivation of $K$ contracture

1) Dependence of inactivation of the degree and the duration of coditioning depolarization. The time-dependence of the inactivation of $\mathrm{K}$ contracture induced by various conditioning depolarizations was measured, according to the procedure described by FrankenHAEUSER and LÄNNERGREN (1967) and SHINOZAKI and OотA (1976). The fibers were bathed in the conditioning solution containing 15,20 or $30 \mathrm{~mm} \mathrm{~K} \mathrm{~K}^{+}$before their exposure to test solution $\left(190 \mathrm{~mm} \mathrm{~K} \mathrm{~K}^{+}\right.$Ringer solution). Under these conditioning depolarizations, the peak tension of the test $\mathrm{K}$ contractures decreased with lengthening of the conditioning time (Figs. 1a, $1 \mathrm{~b}$ and $1 \mathrm{c})$. When the duration of the conditioning depolarization is constant ( $1 \mathrm{~min}$ ), the peak tension of the test $\mathrm{K}$ contracture was reduced depending on the degree of the conditioning depolarization: by pretreatment of the fibers with 15,20 or $30 \mathrm{mM} \mathrm{K}^{+}$, the peak tension of the test $\mathrm{K}$ contracture was inhibited 

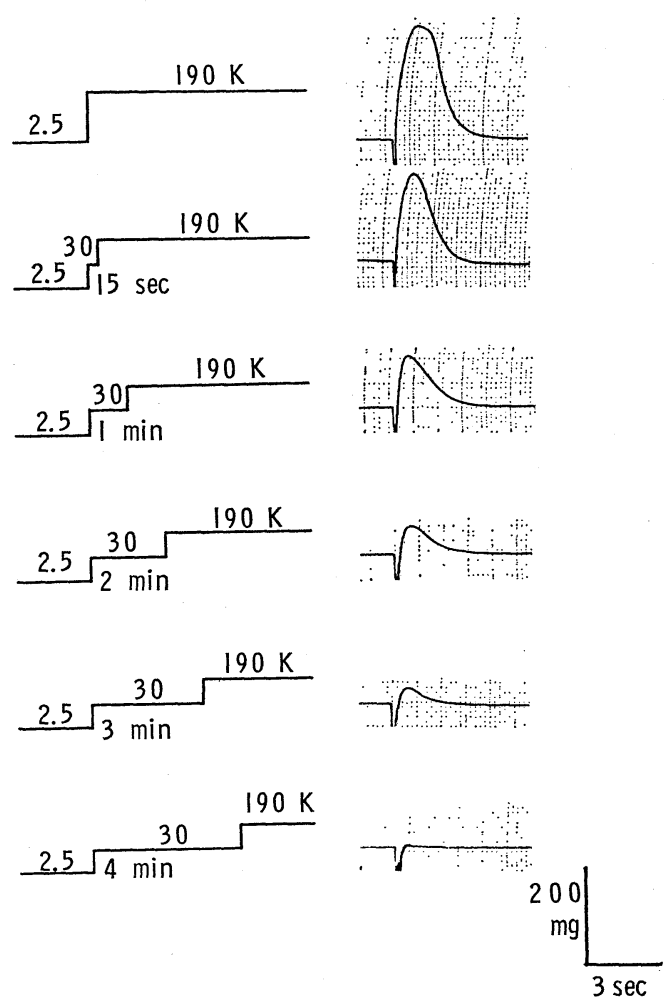

Fig. 1c. The decrease in peak tension of test $\mathrm{K}$ contracture induced by conditioning with $30 \mathrm{~mm} \mathrm{~K} \mathrm{~K}^{+}$. Conditioning solution: choline Ringer solution containing $30 \mathrm{~mm} \mathrm{KCl}$. Other conditions are the same as stated for Fig. 1a.

to 90,68 or $45 \%$, respectively (see also Fig. 2). When these values were plotted against the concentration of $\mathrm{K}^{+}$on the abscissa, a similar inactivation curve to that reported by SHINOZAKI and OoTA (1976) was obtained.

2) Time-dependence of inactivation. Using the values obtained in the foregoing experiments (Figs. 1a, $1 \mathrm{~b}$ and 1c), time-dependence curves of inactivation of $\mathrm{K}$ contracture were drawn (Fig. 2). By the conditioning of a single fiber with $15 \mathrm{mM} \mathrm{K} \mathrm{K}^{+}$, the peak tension of the test $\mathrm{K}$ contracture was very slowly decreased and this inactivation reached a steady level (relative tension, 0.83) at about $3 \mathrm{~min}$ : we term this phase the first phase of inactivation. A similar result was observed in the case of the conditioning depolarization with $10 \mathrm{~mm} \mathrm{~K} \mathrm{~K}^{+}$. The first phase of inactivation induced by $15 \mathrm{mM} \mathrm{K} \mathrm{K}^{+}$further increased very slowly. In the case of conditioning with $20 \mathrm{mM} \mathrm{K} \mathrm{K}^{+}$, the inactivation developed more rapidly during the early stage of the conditioning period and reached a steady level (relative tension, 0.60 ) at about $2 \mathrm{~min}$ (the first phase of inactivation). The inactivation then increased again and finally reached a full inactivation level more slowly: we 


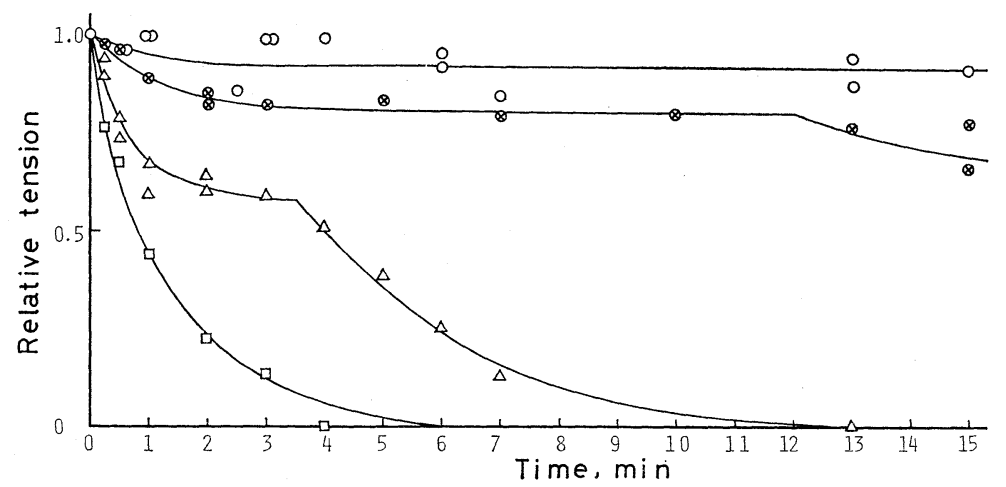

Fig. 2. Time-dependence of the inactivation induced by conditioning depolarization with

$10,15,20$ and $30 \mathrm{~mm} \mathrm{~K}^{+} . \quad, 10 \mathrm{mM} \mathrm{K}^{+} ; \otimes, 15 \mathrm{mM} \mathrm{K}^{+} ; \triangle, 20 \mathrm{mM} \mathrm{K}^{+} ; \square, 30 \mathrm{~mm}$

$\mathrm{K}^{+}$. Conditions are the same as stated for Fig. 1.

term this phase the second phase of inactivation. These results are similar to that recently reported by TAKAUII and NAGAI (1977). In the case of conditioning with $30 \mathrm{mM} \mathrm{K}^{+}$, the time course of inactivation became more rapid and it reached the full level without passing through a steady level.

In summary, the time course of inactivation is biphasic: the first phase reaches a steady level depending on the degree of conditioning depolarization, while the second phase reaches its full inactivation independently of the degree of conditioning depolarization, but the onset of the second phase depends on the degree of the conditioning depolarization.
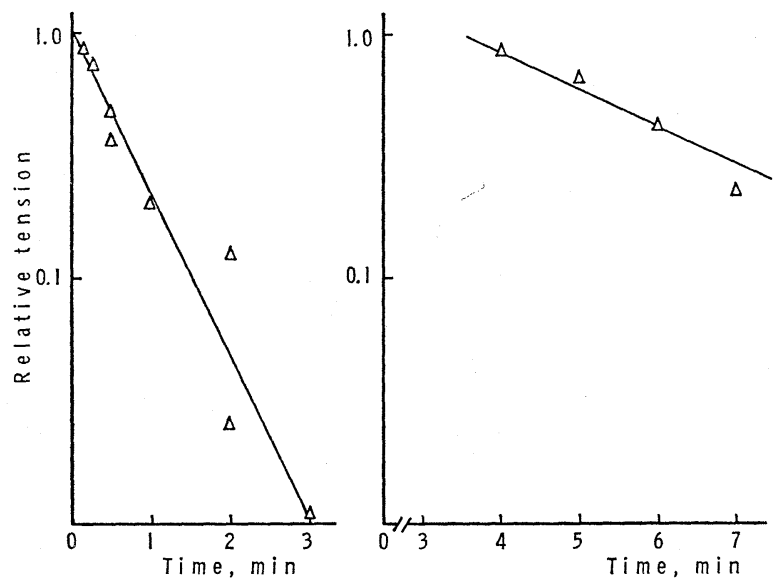

Fig. 3. Analysis on semilogarithmic plot of the time-dependence curve of the inactivation induced by conditioning depolarization with $20 \mathrm{~mm} \mathrm{~K}^{+}$. The first phase and the second phase of inactivation are plotted on the left and on the right, respectively. 1.0 on the ordinate indicates the tension of test contracture at the starting point for the first phase and that at steady level for the second phase. The data points plotted in this figure are the same as those plotted in Fig. 2. 
2. Analysis of the time course of the first and second phases of inactivation

In the present study, it was observed that when the relative tension-time curve obtained with the conditioning depolarization at $20 \mathrm{mM} \mathrm{K} \mathrm{K}^{+}$(Fig. 2) are expressed on a semilogarithmic scale (Fig. 3), the curve consists of two straight lines which correspond to the first and second phases. Therefore, it is evident that the time course of the inactivation of $\mathrm{K}$ contracture is exponential

A

Conditioning at $1.8 \mathrm{mM} \mathrm{Ca}$
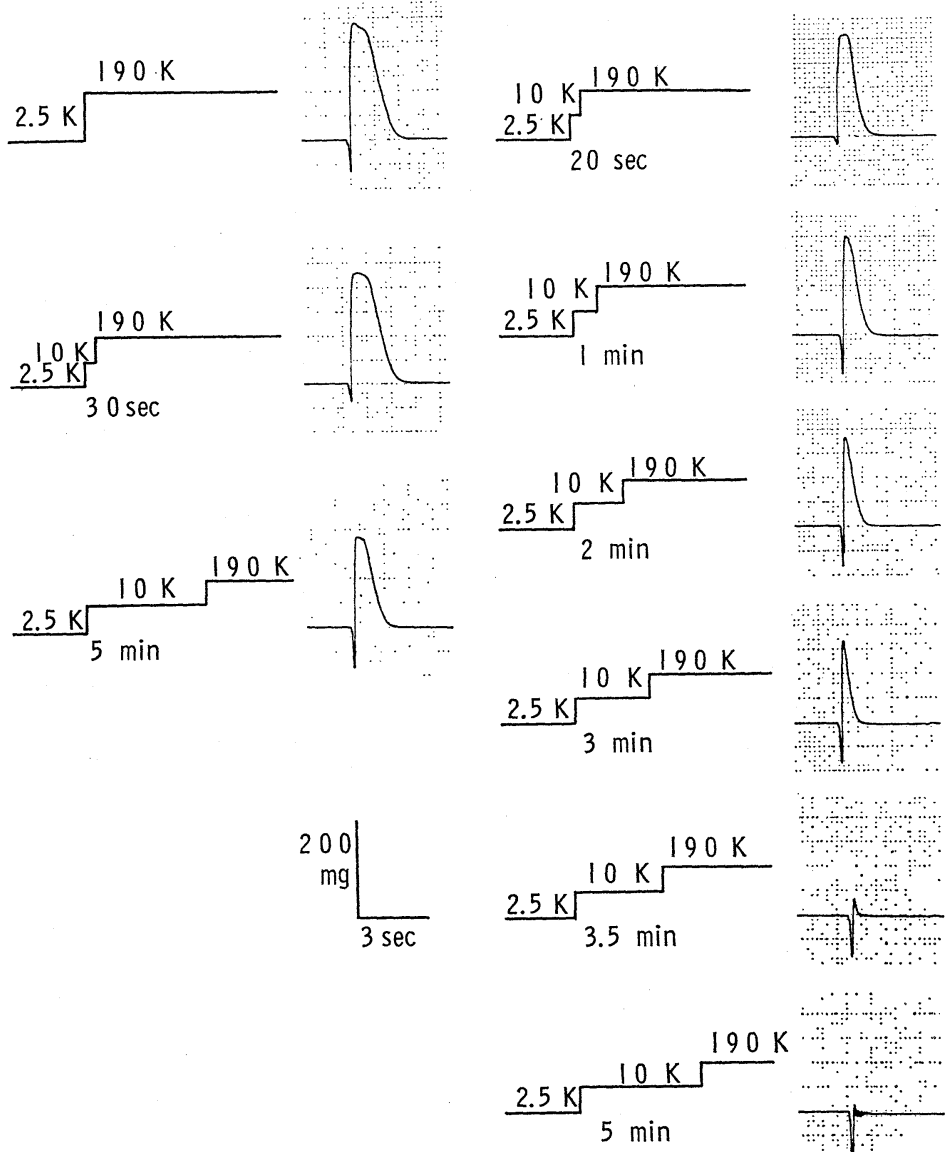
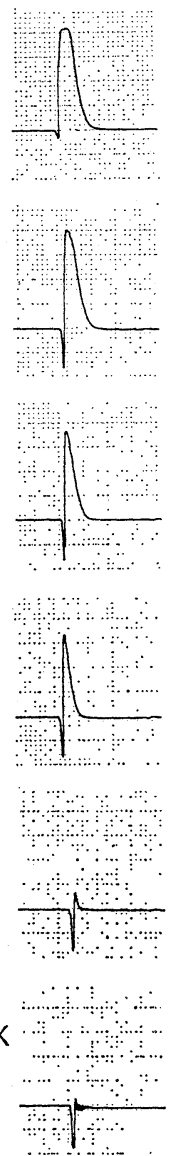

Fig. 4. Effect of low concentration of $\mathrm{Ca}^{2+}$ in the conditioning solution on the peak tension of test $\mathrm{K}$ contracture. A, conditioning with $10 \mathrm{mM} \mathrm{K}^{+}$at $1.8 \mathrm{mM} \mathrm{Ca}$; B, conditioning with $10 \mathrm{mM} \mathrm{K}^{+}$at $0.18 \mathrm{~mm} \mathrm{Ca}$. Note the facilitated inactivation. Test contracture was induced by $190 \mathrm{~mm} \mathrm{~K}^{+}$at $1.8 \mathrm{mM} \mathrm{CaCl}_{2}$. Fiber diameter, $75 \mu \mathrm{m}$. Other conditions are the same as for Fig. 1. 


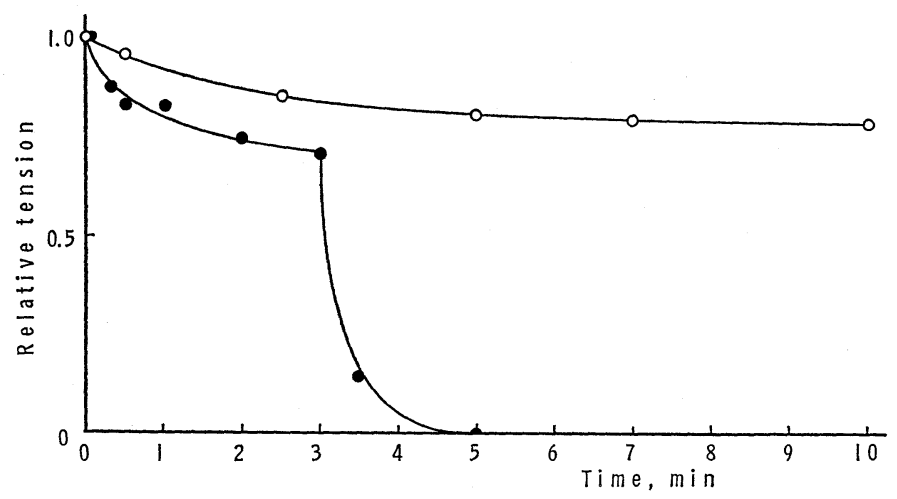

Fig. 5. Effect of low concentration of $\mathrm{Ca}$ on the time-dependence curve of the inactivation induced by conditioning depolarization at $10 \mathrm{mM} \mathrm{K} \mathrm{K}^{+}$. The data points plotted in this figure are the same those described in Fig. 4. $\bigcirc, 1.8 \mathrm{~mm} \mathrm{Ca} ; \bullet, 0.18 \mathrm{~mm} \mathrm{Ca}$.

in both the first and second phases. On the other hand, in the case of conditioning with $30 \mathrm{~mm} \mathrm{~K}^{+}$, the time course of inactivation was monophasic. However, this seems to be a combination of the first and second phases of inactivation, since log tension-time plots indicated that the curve consists of two straight lines (not shown here). From the log tension-time plots, the time constants of the first phase of the inactivation induced by the conditioning depolarization with 15,20 and $30 \mathrm{~mm} \mathrm{~K} \mathrm{~K}^{+}$was 68,40 and $59 \mathrm{sec}$, respectively, and those of the second phase with 20 and $30 \mathrm{~mm} \mathrm{~K}^{+}$was 177 and $27 \mathrm{sec}$, respectively.

\section{Effect of calcium concentration}

Effect of low calcium concentration on the time course of inactivation was examined (Figs. 4 and 5). In the presence of $1.8 \mathrm{mM} \mathrm{Ca}^{2+}$, by conditioning with $10 \mathrm{mM} \mathrm{K}^{+}$, the first phase of inactivation of $\mathrm{K}$ contracture was observed, but not the second (Figs. 4 and 5). By lowering the Ca concentration to $0.18 \mathrm{~mm}$, the time-dependence curve of the inactivation markedly shifted to the left, and a biphasic character appeared. From a similar analysis of this curve to that in Fig. 3, the time course of the inactivation consists of two exponential curves with time constants of 54 and $23 \mathrm{sec}$.

\section{DISCUSSION}

The present study clearly indicates that the time course of the inhibition of $\mathrm{K}$ contracture induced by conditioning depolarization, i.e., the time course of the inactivation is biphasic, consisting of the first phase and the second phase (Fig. 2).

The first phase of the time-dependence curve of the inactivation shows the following characteristics: 1) the time course and the extent of the inactivation depend on the degree of conditioning depolarization; 2) the inactivation of this 
phase exponentially proceeds and finally reaches a steady level; and 3) the rate of the first phase is markedly increased by lowering $\mathrm{Ca}$ in the external medium. These characteristics resemble those of the inactivation of sodium conductance in a squid giant axon (HodgKIN and HuXLEY, 1952a, b; FrankeNHAEuSER and Hodgkin, 1957). Therefore, it can be considered that the first phase of the time-dependence curve of inactivation of $\mathrm{K}$ contracture is an analogical phenomenon with the inactivation of sodium conductance.

The characteristics of the second phase of the inactivation are clearly different in several points from those of the first phase; the second phase of the inactivation reaches its final level (full inactivation) independently of the concentration of $\mathrm{K}$ for conditioning and the time constant is far longer. It seems that the existence of the second phase has not been shown on the inactivation of sodium conductance of squid giant axon. In these circumstances, it is interesting to notice the recent report of MANDRINO (1977), which indicates that the inactivation of the sodium current in frog single skeletal muscle fibers shows two phases.

We previously proposed that the inactivation of $\mathrm{K}$ contracture consists of two distinct processes, and tentatively termed these processes inactivation 1 and inactivation 2 (Kosaka et al., 1977; TAKaujI and NAGAI, 1977). According to our previous view, inactivation 1 was related to only the spontaneous relaxation of $\mathrm{K}$ contracture, while inactivation 2 was related to only the inhibition of the peak contracture tension in the whole range of the time-dependence curve of the inactivation. However, the inhibition of peak tension induced by inactivation implies the inhibition of activation by some process which is caused by depolarization with $\mathrm{K}$, and the inactivation related to the spontaneous relaxation also involves the inhibition of activation induced by a similar process. Therefore, it may be unreasonable to distinguish the inactivation related to spontaneous relaxation from that related to the inhibition of peak tension. This consideration could be supported by the fact that the inactivation of sodium conductance influences the rate of falling phase of action potential (HodGKIN and HuXLEY, 1952a, b). As mentioned above, the first phase of the time course of mechanical inactivation shown in Fig. 2 has an analogical character with the inactivation of sodium conductance. This result suggests that the first phase of the inactivation influences the rate of spontaneous relaxation of $\mathrm{K}$ contracture, since the inactivation of sodium conductance affects the rate of falling phase of action potential, and this, in turn, suggests that the first phase of the inactivation includes the above-mentioned inactivation 1 previously proposed by us (KosAKA et al., 1977; TAKAUJI and NAGAI, 1977). Hence, it would be reasonable to apply the term inactivation 1 to the first phase of inactivation of $\mathrm{K}$ contracture. On the other hand, since the second phase of inactivation is at present considered to have a different character from the first phase (see above), we apply the term inactivation 2 to the second phase of inactivation of $\mathrm{K}$ contracture. Thus, the terms inactivation 1 and inactivation 2 are finally given to the first phase 
and the second phase of inactivation (Fig. 2), respectively, with a partly different meaning from that of the terms originally proposed.

LÜTTGAU (1963) described mechanical inactivation as being analogical to the inactivation of sodium conductance. From the foregoing discussion, this analogy should be limited to the first phase of mechanical inactivation (inactivation 1) in our present paper. On the other hand, FrankenHAEUSER and LÄNNERGREN (1967) pointed out that the inactivation induced by conditioning depolarization with a subthreshold concentration of $\mathrm{K}$ reaches full inactivation without passing through a steady level. This inactivation is considered to correspond mainly to the second phase of inactivation (inactivation 2) in our present paper.

It has been reported that the inhibition of peak tension of the test contracture with $190 \mathrm{mM} \mathrm{K}^{+}$is relatively small, immediately after the spontaneous relaxation of the contracture induced by application of 30 or $40 \mathrm{mM} \mathrm{K} \mathrm{K}^{+}$(FrANKENHAEUSER and LäNNERgren, 1967; Stuesse and Lindley, 1975; KosaKa et al., 1977). From our view mentioned above, on the biphasic time course of inactivation and the final expression of inactivation 1 and inactivation 2, this phenomenon may be explained by considering that during the spontaneous relaxation phase of the contracture induced by conditioning with a moderate concentration of $\mathrm{K}$, inactivation 1 (the first phase of inactivation) has already fully occurred, but inactivation 2 (the second phase of inactivation) has not yet occurred sufficiently.

Frankenhaeuser and LänNergren (1967), StuesSE and Lindley (1975) and KosAKA et al. (1977) have emphasized that in the case of conditioning depolarization with subthreshold concentrations of $\mathrm{K}$, the inactivation of $\mathrm{K}$ contracture occurs without a preceding activation. This may mean that the threshold for the inactivation process (consisting of inactivations 1 and 2) of $\mathrm{K}$ contracture is lower than that for the activation process. However, the fact that the inactivation process (involved in the spontaneous relaxation) of a contracture, induced by superthreshold concentrations of $\mathrm{K}$, follows the activation process may imply that the rate of development of the activation process is larger than that of the inactivation process under this condition. The "inactivation without a preceding activation" mentioned above also has been observed in the case of sodium conductance (HoDGKIN and HuXLEY, 1952a).

In the present experiment (Figs. 4 and 5), it was demonstrated that at $1.8 \mathrm{~mm}$ $\mathrm{Ca}$ and $10 \mathrm{~mm} \mathrm{~K}$, the time-dependence curve of the inactivation was monophasic (within at least $10 \mathrm{~min}$ ) and had a time constant of $90 \mathrm{sec}$ and, on the other hand, at low $\mathrm{Ca}$ concentration $(0.18 \mathrm{~mm})$ the curve showed a biphasic character: the first phase (inactivation 1) and the second phase (inactivation 2) had time constants of 54 and $23 \mathrm{sec}$, respectively. These results indicate that both inactivation 1 and inactivation 2 are facilitated by the lowering of $\mathrm{Ca}$ in the external medium. 


\section{REFERENCES}

Frankenhaeuser, B. and Hodgkin, A. L. (1957) The action of calcium on the electrical properties of squid axons. J. Physiol. (Lond.), 137: 218-244.

Frankenhaeuser, B. and LänNergren, J. (1967) The effect of calcium on the mechanical response of single twitch muscle fibres of Xenopus laevis. Acta Physiol. Scand., 69: 242-254.

Hodgkin, A. L. and Horowicz, P. (1959) The influence of potassium and chloride ions on the membrane potential of single muscle fibres. J. Physiol. (Lond.), 148: 127-160.

Hodgkin, A. L. and Huxley, A. F. (1952a) The dual effect of membrane potential on sodium conductance in the giant axon of Loligo. J. Physiol. (Lond.), 116: 497-506.

Hodgkin, A. L. and HuXLEY, A. F. (1952b) A quantitative description of membrane current and its application to conduction and excitation in nerve. J. Physiol. (Lond.), 117: 500-544.

Kosaka, I., Oota, I., Suzuki, T., and Nagai, T. (1977) Time- and Na-dependent effects of $\mathrm{Ca}$ depletion on potassium contracture in frog twitch muscle fiber. Jpn. J. Physiol., 27: 511-524.

LÜTTGAU, H. C. (1963) The action of calcium ions on potassium contractures of single muscle fibres. J. Physiol. (Lond.), 168: 679-697.

MandRino, M. (1977) Voltage-clamp experiments on frog single skeletal muscle fibres: Evidence for a tubular sodium current. J. Physiol. (Lond.), 269: 605-625.

SHINOZAKI, F. and Oota, I. (1976) Potassium-induced contracture in frog twitch muscle fiber: Relation between mechanical activation and inactivation. Sapporo Med.J., 45:41-50 (in Japanese).

Stuesse, S. C. and Lindley, B. D. (1975) Contractile inactivation in frog single denervated muscle fibers. Am. J. Physiol., 229: 1492-1497.

TAKAUJI, M. and NAGAI, T. (1977) Effect of dantrolene sodium on the inactivation of excitation-contraction coupling in frog skeletal muscle. Jpn. J. Physiol., 27: 743-754.

Takauj, M., Kosaka, I., Tsutsu-ura, M., and NagaI, T. (1978) Biphasic time course of the inactivation of potassium contractures in single twitch muscle fibers of the frog. Abstracts of the VIth International Biophysics Congress Kyoto, Japan, p. 235. 